\title{
O Estado da Arte da Pesquisa em Prevenção em Saúde Mental no Brasil: uma Revisão Sistemática
}

\author{
Samia Abreu \\ Universidade de Brasília \\ Sheila Giardini Murta \\ Universidade de Brasília
}

\begin{abstract}
RESUMO
Realizou-se uma revisão sistemática de literatura sobre a pesquisa em prevenção no Brasil conforme as etapas do ciclo de investigação na área: análise do problema, identificação de fatores de risco e proteção, intervenção-piloto, teste avançado, avaliação de efetividade e difusão. Foram encontrados 4.131 artigos, dos quais $31(0,75 \%)$ descreviam programas preventivos e 11 destes $(35,4 \%)$ eram sistematicamente avaliados, com a foco em prevenção de comportamento antissocial, rejeição por pares, violência e abuso de substâncias. Os estudos se concentram nos estágios iniciais do ciclo proposto, praticamente inexistindo estudos nos estágios finais. Recomendam-se desenvolvimento de medidas de avaliação, interlocução entre prevenção e promoção de saúde mental, fortalecimento de rede de pesquisadores e intercâmbio com políticas públicas para avanço da área.
\end{abstract}

Palavras-chave: Saúde mental; prevenção; políticas públicas; promoção de saúde.

\begin{abstract}
The state of the art of the research in mental health prevention in Brazil: A systematic review

It was done a systematic literature review to describe the state-of-the-art on mental health prevention in Brazil according to the stages of the preventive research cycle: analysis of the problem, risk and protective factors identification, pilot intervention, advanced test, effectiveness evaluation and diffusion. We found 4131 articles of which $31(0.75 \%)$ targeted on preventive programs and only $11(35.4 \%)$ were systematically evaluated; the prevention focus of the programs were on antisocial behavior, peers rejection, violence and substance abuse. The studies are concentrated in the early stages of the research cycle, barely inexistent studies in the final stages. It is recommended the development of evaluation measures, dialogue between prevention and health promotion, strengthening of researches network and exchange with public policies for the progress of this field in Brazil.
\end{abstract}

Keywords: Mental health; prevention; public policies; health promotion.

Ao redor do mundo, pesquisadores têm investigado como prevenir desfechos negativos em saúde mental e encontrado resultados positivos. Há evidências da eficácia e efetividade de programas preventivos para vários fatores de risco, tais como maus-tratos parentais (Durlak \& Wells, 1997; Knerr, Gardner, \& Cluver, 2013) e violência no namoro (Murta, Santos, Martins, \& Oliveira, 2013), e desfechos negativos em saúde mental, como transtornos de depressão (Christensen, Pallister, Smale, Hickie, \& Calear, 2010; Cuijpers, Straten, Smit, Mihalopoulos, \& Beekman, 2008; Muñoz, Cuijpers, Smit, Barrera, \& Leykin, 2010) e ansiedade (Christensen et al., 2010; Fisak Jr, Richard, \& Mann, 2011).

No Brasil, estima-se que $3 \%$ da população brasileira sofra de algum transtorno mental severo e persistente e necessite de cuidados contínuos em saúde mental. Ademais, $9 \%$ da população utiliza atendimentos eventuais em saúde mental (Brasil, Ministério da Saúde, Departamento de Ações Programáticas Estratégicas, Coordenação de Saúde Mental, 2003). Investimentos no tratamento de pacientes com sofrimento psíquico grave têm sido crescentes no Brasil (Brasil, Ministério da Saúde, Departamento de Ações Programáticas Estratégicas, Coordenação de Saúde Mental, 2006). Por outro lado, esforços para a ampliação de serviços em atenção básica e a promoção de saúde mental têm sido também solicitados pela sociedade, conforme evidenciado pela recente Conferência Nacional de Saúde Mental (Sistema Único de Saúde, Conselho Nacional de Saúde, \& Comissão Organizadora da IV Conferência Nacional de Saúde 
Mental, 2010). Embora o investimento na oferta de tratamento seja reconhecidamente urgente e relevante, destaca-se, igualmente, a necessidade de prevenção e intervenção precoce para aqueles que estão expostos a fatores de risco ou que apresentem sintomas iniciais de uma desordem mental, para que não adoeçam gravemente e tampouco dependam de tratamentos dispendiosos e equipes especializadas, nem sempre disponíveis.

Para além da redução do sofrimento e deperdas diversas para indivíduos e famílias, os investimentos em prevenção são altamente vantajosos do ponto de vista econômico, tomando-se como unidades de análise os benefícios e custos associados. Achados do departamento de saúde britânico resultantes de análises econômicas demonstraram que o investimento de uma libra esterlina em programas preventivos e de promoção de saúde resulta na economia de 83 libras esterlinas à saúde do país (Knapp, McDaid, \& Parsonage, 2011). Portanto, as evidências sugerem que investimentos em pesquisas no âmbito da prevenção em saúde mental são indicados, seja do ponto de vista ético, social ou econômico.

A robustez desses resultados em avaliação de eficácia, efetividade e custo-efetividade de programas preventivos se apoia numa ampla cadeia de produção de conhecimentos na área, já consolidada no hemisfério Norte há décadas (Durlak \& Wells, 1997; Lacerda \& Guzzo, 2005). O ciclo de pesquisa em prevenção em saúde mental pode ser organizado em seis grandes etapas: a identificação do problema e sua prevalência; a localização de seus fatores de risco e proteção; o testepiloto da intervenção preventiva; o teste de eficácia; o teste de efetividade; e a difusão (Dumka, Roosa, Michaels, \& Suh, 1995). A primeira etapa está associada a estudos epidemiológicos, quando o problema que se busca prevenir é descrito em suas manifestações, prevalência e incidência. A segunda etapa recai sobre a investigação das condições, pessoais e contextuais, que aumentam a vulnerabilidade da população-alvo (fatores de risco) e das que minimizam os fatores de risco e protegem a saúde mental (fatores de proteção). Esses achados guiam o planejamento posterior de programas preventivos, com implicações para a definição dos objetivos e componentes das intervenções. A terceira etapa consiste no desenvolvimento de um programa, baseado em teoria, seguido de um teste-piloto. Decisões para aprimoramento de procedimentos e estratégias de intervenção e avaliação são tomadas nessa etapa. A quarta etapa compreende o teste avançado para a análise da eficácia do programa, por meio de estudos experimentais, amostras amplas, medidas confiáveis e válidas e avaliações de seguimento (Flay, Biglan, Boruch, Castro, Gottfredson, Kellam, Moscicki, Schinke, Valentine, \& Ji, 2005). A quinta etapa direciona-se à implementaçãodo programa em novos contextos por multiplicadores treinadospelos pesquisadores, a fim de se avaliar a validade externa dos resultados. A manualização do programa, a capacitação de gestores e facilitadores, medidas para recrutamento e retenção dos participantes e cuidados com a fidelidade da implementação são aspectos relevantes nessa etapa (Flay et al., 2005). O último estágio é a difusão, quando programas eficazes e efetivos são adotados, implementados e disseminados em comunidades, regiões ou países inteirose tornam-se acessíveis em larga escala, podendo ou não se sustentar ao longo do tempo (Tibbits, Bumbarger, Kyler, \& Perkins, 2010) e impactar a saúde mental da população.

No Brasil, nos últimos anos, programas preventivos com eficácia e efetividade testadas e que estejam prontos para ser difundidos em serviços previstos nas políticas públicas têm sido gradualmente mais requeridos. Essa demanda pode ser observada no expressivo aumento de políticas públicas federais de cunho preventivo, estabelecidas por diferentes ministérios. Por exemplo, o Ministério da Saúde (Tykanori, Santos, Pedroso, Abreu, Rabbani, Rocha, Schneider, Sanchez, Atayde, Medeiros \& Scafuto, 2013), associado ao Ministério do Desenvolvimento Social e Combate à Fome, em parceria com o Escritório das Nações Unidas sobre Drogas e Crime (UNODC), vem adaptando para a cultura brasileira programas de prevenção primária ao abuso de álcool e drogas, embasados em evidências de efetividade e dirigidos ao fortalecimento de competência social em crianças e adolescentes e à promoção de relações familiares positivas. O Ministério do Desenvolvimento Social e Combate à Fome vem desenvolvendo o Serviço de Proteção e Atendimento Integral à Família (PAIF) (Brasil, Ministério do Desenvolvimento Social e Combate à Fome, 2012), focado no acompanhamento proativo, protetivo, preventivo e territorializado de famílias em situação de vulnerabilidade socioeconômica. A Política Nacional de Atenção Integral à Saúde do Homem (Brasil, Ministério da Saúde, Secretaria de Atenção à Saúde, 2008) prevê ações educativas para prevenção da violência e promoção de direitos sexuais e reprodutivos como um 
dos focos prioritários de atenção na saúde do homem, incluindo o homem adolescente.

Apesar dessa demanda crescentenas políticas públicas, publicações da última década indicam ser pequena a produção brasileira de pesquisas de desenvolvimento e avaliação de programas de prevenção em saúde mental (Benetti, Ramires, Schneider, Rodrigues, \& Tremarin, 2007; Murta, 2007). Em particular, são poucas as revisões de literatura que têm como tema principal os programas preventivos em saúde mental desenvolvidos no Brasil. Revisões de literatura, sobretudo sistemáticas ou metanálises, constituem o primeiro passo para uma descrição acurada de evidências de eficácia e efetividade de um dado tipo de intervenção, bem como de suas forças e vulnerabilidades. Seus achados podem guiar agendas de pesquisa e subsidiar a tomada de decisão por parte de pesquisadores, profissionais, formuladores de políticas públicas e gestores em saúde (Melnik \& Atallah, 2011).

As revisões de literatura existentes (Luizzi \& Rose, 2010; Pelisoli \& Piccoloto, 2010; Silva \& Salles, 2010; Rios \& Williams, 2008; Louzã, 2007; Murta et al., 2013) descrevem ou avaliam, prioritariamente, programas internacionais, exceto por dois estudos com foco exclusivo na produção nacional. As exceções são um estudo a respeito do uso de treinamento de habilidades sociais aplicado à prevenção primária, secundária e terciária (Murta, 2005) e outro sobre os programas de prevenção ao consumo de drogas (Canoletti \& Soares, 2004).

Considerando a lacuna em revisões sistemáticas de literatura em programas de prevenção em saúde mental no Brasil e a relevância social dessa temática no atual cenário das políticas públicas em saúde mental, realizou-se uma revisão sistemática da produção nacional na área para descrever o estado da arte da pesquisa em prevenção em saúde mental no Brasil. Especificamente, buscou-se descrever os temas que caracterizam a produção nacional em prevenção em saúde mental, identificar os estudos relativos aos programas de prevenção primária em saúde mental sistematicamente avaliados e situar em que etapa se encontram os estudos nacionais conforme as fases do ciclo de pesquisa em prevenção, previamente descritas.

\section{MÉTODO}

Para proceder à revisão sistemática de literatura nacional, foram identificados artigos científicos sobre prevenção e analisados os estudos cujo tema principal era a prevenção em saúde mental. Foram utilizadas as bases de dados SciELO e PePSIC, por serem os principais indexadores nacionais de publicações em saúde mental. Não houve limite inicial de data para publicação. Foram incluídos artigos publicados até janeiro de 2012.

\section{Procedimentos de seleção dos estudos}

Foram utilizadas as seguintes palavras-chaves: "prevenção" or "atenção primária" or "atenção básica" or "promoção de saúde". Inicialmente, todos os títulos dos estudos encontrados passaram por uma leitura flutuante (Bardin, 1977). Esta consiste em um primeiro contato com o material de análise e levantamento das primeiras categorias que seriam definidas conforme os focos de prevenção observados. A partir dessa primeira análise, os estudos encontrados na busca por palavraschave foram organizados em estudos de prevenção de: (a) doença mental; (b) doença física; (c) acidentes; (d) adversidades ambientais; (e) doenças em animal; e (f) problemas agrícolas. Em caso de dúvida, na classificação dos estudos, era solicitado o parecer final de um juiz, pesquisador da área de prevenção em saúde mental.

Os estudos acerca de "doença mental" incluíam artigos com foco primário na prevenção de um transtorno mental (desfecho final), conforme classificação do Manual Diagnóstico e Estatístico de Transtornos Mentais - 4a Edição (DSM-IV), como transtornos alimentares, de ansiedade e depressão, ou um fator de risco para transtornos mentais (desfecho intermediário), como violência, uso de álcool, uso de drogas, abuso sexual e dificuldades escolares. A categoria "doença física" inclui, por exemplo, prevenção a doenças diversas, como doenças respiratórias, doenças odontológicas e doenças crônicas, e fatores de risco em saúde física, como comportamento sexual de risco e tabagismo. A categoria "acidentes" incluiu eventos automobilísticos, quedas domésticas, queimaduras, acidentes biológicos e acidentes de trabalho. A categoria "adversidades" teve como foco os estudos sobre a prevenção de desastres ambientais e geotecnologia, como inundações, incêndios e derramamentos de óleo. "Doenças em animais" incluíram artigos que descreviam a prevenção de doenças cardiovasculares, respiratórias e digestivas e intoxicação de animais, e "problemas agrícolas" abrangeram efeitos do uso de herbicidas, interferência de plantas daninhas e prevenção de insetos e predadores em plantações. 
Para os estudos com títulos não específicos, ampliou-se a busca à leitura do resumo. Os resumos cujo objetivo principal não era a prevenção de nenhuma das condições citadas anteriormente foram lidos e criaram-se mais cinco categorias de análise. Os focos das novas categorias foram: (g) discussão saúde e doença; (h) formação, com artigos sobre a formação de profissionais de saúde (enfermeiro, fisioterapeuta, fonoaudiólogo, nutricionista, médico) e de outras áreas (sociologia, antropologia) em face da atuação preventiva; (i) influência da cultura, com estudos teóricos sobre a concepção de saúde e influência cultural em comportamentos saudáveis e não saudáveis; (j) papel do SUS (Sistema Único de Saúde) e seus setores, como por exemplo, o Programa de Saúde da Família e a Unidade Básica de Saúde, com a discussão sobre suas ações políticas, sociais e econômicas; (k) direitos humanos e aplicabilidade das leis em relação à saúde; (1) prevenção de fraude, corrupção e temáticas relacionadas a política, segurança pública e direitos do cidadão; e (m) estudo de método ou procedimento de pesquisa, como ensino e discussão de revisões sistemáticas, práticas baseadas em evidências e etnografia.

Os artigos identificados na categoria "doença mental" passaram por uma análise detalhada do resumo. Em muitos estudos, a leitura do resumo não foi suficiente para definir o foco, sendo então realizada a leitura do artigo completo. Os artigos cujo foco não era prevenção e os estudos estrangeiros cujos autores e/ou o local de origem da pesquisa não era o Brasil foram contabilizados e excluídos. Foram também eliminados estudos duplicados e resenhas, editoriais, opinião ou qualquer outra seção da revista que não fosse um artigo científico.

\section{Critérios de análise de estudos de prevenção em saúde mental}

As publicações em prevenção em saúde mental que compuseram a amostra final (374 artigos) foram analisadas conforme as categorias: 1) prevenção primária (estudos que descreviam intervenções de prevenção primária em saúde mental, estudos de avaliação de necessidades, de capacitação de profissionais para a atuação em programas preventivos, de relatos de experiências com intervenções preventivas e descrições de ações implantadas como políticas de prevenção); 2) prevenção secundária ou terciária (estudos teóricos com foco na prevenção de danos resultantes de um transtorno mental já diagnosticado);
3) promoção de saúde mental (discussão teórica ou conceitual sobre a promoção de saúde mental ou programa de promoção de saúde mental, bem-estar e qualidade de vida, sem especificação de variáveis prevenidas); 4) fundamentação (estudos teóricos, conceituais, de pesquisa básica, epidemiológicos ou de revisão de literatura sobre prevenção); 5) material didático e recursos (descrição e/ou avaliação de cartilhas, manuais ou jogos para prevenção em saúde mental); 6) políticas públicas (estudos de apresentação ou análise de efeitos de políticas públicas relacionadas a condições específicas de prevenção); e 7) avanços e limitadores (discussão sobre os desafios e avanços da pesquisa em prevenção e para a implantação e avaliação de programas preventivos). Essas categorias foram geradas por dois pesquisadores. Em caso de divergência, um juiz era solicitado, até que refinamentos sucessivos permitissem a definição final da categoria.

Por fim, as publicações na categoria "prevenção primária" (31 artigos) foram analisadas para identificação de estudos de avaliação sistemática de programas preventivos, de acordo com os critérios de Doughty (2005): delimitação clara da(s) variável(is) avaliada(s) na intervenção, análise de uma variável que indicasse um desfecho em saúde mental, descrição de medidas qualitativas e/ou quantitativas específicas para mensurar a variável citada eapresentação de resultados.

\section{Cálculo de concordância entre codificadores}

As análises dos estudos da amostra final (374 artigos) foram feitas por dois codificadores independentes. Foram comparadas as análises entre codificadores, mensurando-se o cálculo de concordância entre eles (Kazdin, 1982), ou seja, o número de concordâncias dividido pela somatória de concordâncias e discordâncias e multiplicado por 100 . O grau de concordância encontrado foi de $97,37 \%$.

\section{RESULTADOS}

Inicialmente, foram encontrados 4.131 artigos que citavam a prevenção como sua temática de discussão. Destes, 3.016 eram estudos de prevenção de doença física e 651 destacavam a prevenção de uma condição de saúde mental. Os resultados indicam, portanto, um número restrito de estudos em saúde mental $(15,75 \%)$, em comparação à produção relativa à doença física (73\%). Dentre os 651 estudos centrados em saúde mental, o foco não era a prevenção em cerca de um 
quarto $(24,57 \%)$ deles, isto é, 160 estudos. Eram estudos que descreviam o tratamento de um transtorno mental diagnosticado ou pesquisas que somente citavam a palavra prevenção, mas não havia discussão ou ênfase no tema. Esses estudos eram caracterizados por artigos que finalizavam seus resumos ou suas considerações finais com frases enfatizando a necessidade de investimentos em prevenção.

$\mathrm{O}$ número de estudos em que o tema centralera a prevenção em saúde mental foi reduzido a 374, após a exclusão de 160 artigos cujo foco não era prevenção, 26 artigos duplicados, 59 estudos estrangeiros e 31 ensaios, resenhas e editoriais. Os 374 estudos representaram apenas $9,05 \%$ do total de artigos encontrados. Destes, conforme evidencia a figura 1 , a categoria de maior frequência foi a dos estudos de fundamentação $(68,45 \%)$. Em seguida, foram encontrados os estudos de prevenção primária $(8,29 \%)$, promoção de saúde $(7,71 \%)$, prevenção secundária ou terciária $(5,88 \%)$, políticas públicas $(4,81 \%)$, avanços e limitadores relativos à implantação de programas preventivos $(1,87 \%)$, instrumentos de avaliação $(1,6 \%)$ e materiais e recursos para uso em prevenção $(1,34 \%)$.

A categoria de frequência mais elevada, relativa a estudos de fundamentação, inclúa publicações referentes a estudos teóricos ou conceituais; formação profissional e inserção do psicólogo no SUS ou em práticas voltadas para a prevenção; pesquisa básica, incluindo estudos sobre crenças, representações sociais e percepção de uma população-alvo sobre uma condição em saúde mental a ser prevenida; estudos epidemiológicos, para avaliação de incidência ou prevalência de fatores de risco ou transtornos mentais; estudos para identificação de fatores de risco e proteção e revisão de literatura, narrativas, sistemáticas, ou do estado da arte, sobre conceitos e fatores de risco e proteção. A quantidade de estudos de fundamentação $(69,46 \%)$ é cerca de oito vezes maior do que o número de estudos sobre intervenções preventivas $(8,29 \%)$. Dentre os estudos de fundamentação, a maior ocorrência foi de estudos de prevenção à violência $(35,78 \%)$, seguidos por estudos de prevenção ao uso de drogas $(27,06 \%)$ e uso de álcool $(16,05 \%)$. Entretanto, quando agrupados, os estudos de prevenção ao uso de drogas e álcool passam a representar $51,83 \%$ do total. Há também, nos estudos de fundamentação, uma quantidade expressiva de publicações (19 artigos) sobre a inserção do psicólogo no SUS, a interlocução entre psicologia e saúde coletiva e a clínica ampliada. Todos narram a história inicial da psicologia baseada no modelo biomédico de atendimento individual até as demandas atuais de atuação comunitária e preventiva, com a sua inserção no SUS. Os artigos destacam a necessidade de uma formação em psicologia que ofereça suporte às demandas recentes de atuação preventiva.

Foram identificados 31 artigos acerca de programas de prevenção primária, os quais incluíram intervenções de prevenção primária em saúde mental, estudos de avaliação de necessidades, de capacitação de profissionais para a atuação em programas preventivos, de relatos de experiências com intervenções preventivas e descrições de ações implantadas como políticas de prevenção. Dentre as publicações nessa categoria, foram identificadas apenas $11(2,94 \%)$ relativas a intervenções com avaliação sistemática de resultados. Nesses estudos, as condições prevenidas foram comportamento antissocial (Rodrigues, Dias, \& Freitas, 2010; Bolsoni-Silva, Silveira, \& Ribeiro, 2008; Borges $\&$ Marturano, 2009; Pinheiro, Haase, Prette, Amarante, \& Del Prette, 2006; Murta et al., 2009), rejeição por pares (Castro, Melo, \& Silvares, 2003), violência (Padovani, Schelini, \& Williams, 2009; Prada \& Williams, 2007), abuso de álcool e outras drogas (Micheli, Fisberg, \& Formigoni, 2004; Ronzani, Rodrigues, Batista, Lourenço, \& Formigoni, 2007) e problemas emocionais e comportamentais em crianças (Lohr, Pereira, Andrade, \& Kirchner, 2007).

Os estudos de promoção de saúde mental identificados (29 publicações) descreviam aspectos conceituais e intervenções para promoção de competências associadas à saúde mental. Diferentemente dos programas preventivos, os programas de promoção não especificavam a condição prevenida (fator de risco, como maus-tratos parentais, ou desfecho final, como depressão) nem apresentavam medidas de avaliação da condição prevenida. As condições promovidas mais recorrentes foram habilidades sociais $(72,72 \%)$, seguidas por habilidades de vida $(9,09 \%)$, conhecimento acerca de consequências do uso de álcool e outras drogas $(9,09 \%)$ e sentido da vida $(9,09 \%)$.

Os estudos de prevenção secundária ou terciária (22 publicações), que se caracterizavam pela prevenção do agravamento de um transtorno mental diagnosticado, eram artigos teóricos de prevenção de recaída, redução de danos, intervenção precoce em psicoses e prevenção do suicídio de pacientes com algum transtorno mental estabelecido. Os dados evidenciam um pequeno 


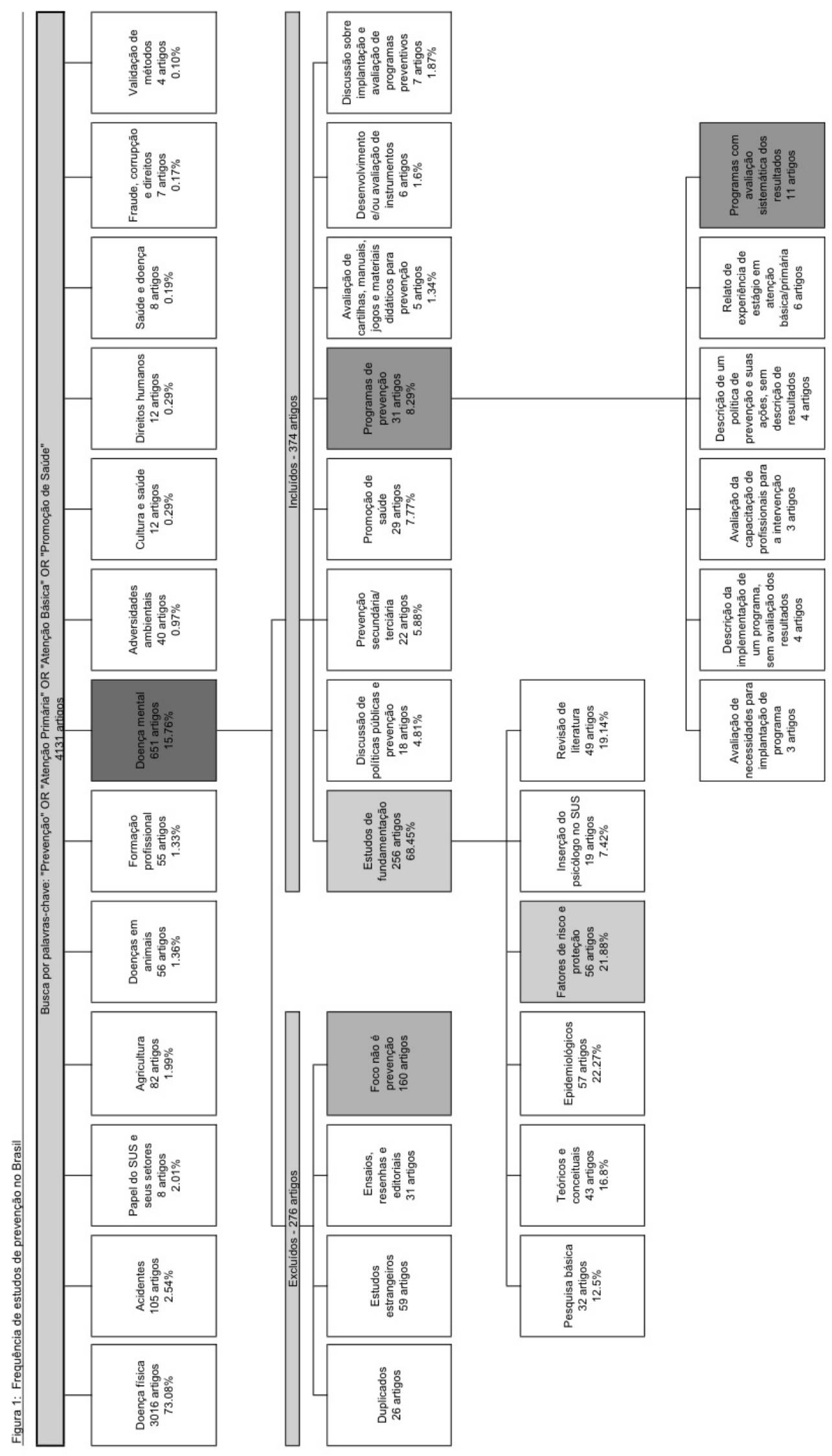


número de publicações cujo foco é política pública em prevenção em saúde mental (4,81\%), produção de instrumentos $(1,6 \%)$ e manuais e recursos para uso em programas preventivos $(1,34 \%)$, conforme demonstra a figura 1 .

Quando os estudos são analisados por etapas do ciclo de pesquisa em prevenção, à medida que o grau de complexidade das etapas do ciclo de pesquisa avança, o número de estudo decresce abruptamente (figura 2). As duas primeiras etapas (caracterização do problema e prevalência e identificação de fatores de risco e proteção) juntas representam $82,09 \%$ de todos os artigos que compõem o ciclo da pesquisa em prevenção no Brasil. Esses estudos se ocupam do levantamento da incidência ou prevalência do problema-alvo (57 artigos), identificação de fatores de risco e proteção (56 estudos), discussão de conceitos e teorias (43 estudos) e verificação de crenças ou representações sociais de uma população em face de uma condição de saúde mental (32 artigos).

Foram identificados 34 artigos focados no desenvolvimento de programas preventivos, cujo alvo era o teste-piloto do programa, por meio de delineamentos não experimentais, e outras atividades típicas dessa etapa, como desenvolvimento de instrumentos e avaliação de necessidades para posterior desenvolvimento do programa. Observa-se na figura 2 que a ocorrência por data de publicação indica um número crescente das primeiras etapas nos últimos seis anos. Enquanto é vasta a produção nas etapas inicias do ciclo de pesquisa em prevenção, ela é escassa nas etapas finais. Foram identificados apenas quatro estudos de avaliação de eficácia e três estudos de avaliação de efetividade. Foi ausente a publicação de artigos que descrevessem a difusão de programas, seja em serviços, seja em políticas públicas.

\section{DISCUSSÃO}

Os resultados indicam que, se comparada à prevenção de doenças físicas, a produção nacional em prevenção em saúde mental é pequena, e grandemente caracterizada por estudos de fundamentação. Poucas pesquisas têm se ocupado de prevenção primária e, dentre estas, um número inexpressivode programas preventivos apresenta avaliação sistemática de resultados. Estes são focados, majoritariamente, na prevenção de fatores de risco para transtornos mentais, como comportamento antissocial, rejeição por pares e violência, e uma menor parte, em desfechos finais, como abuso de álcool e drogas e problemas emocionais e comportamentais. Ademais, os estudos nacionais concentram-se nas etapas iniciais do ciclo de pesquisa em prevenção, como os estudos para descrição de prevalência de transtornos mentais e identificação e seus fatores de risco, seguidos por estudos para testepiloto de programas desenvolvidos. São escassos os estudos de avaliação de eficácia e efetividade e inexistentes os estudos de difusão de programas preventivos baseados em evidências de efetividade. É evidente, portanto, uma descontinuidade na transformação de pesquisa básica em intervenções e tecnologias preventivas, indicada pela ocorrência de cerca de oito vezes mais estudos teóricos em relação ao número de programas. Outra evidência é o crescimento das etapas 1 (identificação de problemas e prevalência) e 2 (identificação de fatores de risco e proteção) do ciclo de pesquisa nos últimos seis anos, diante da ausência de estudos na última etapa (difusão de programas preventivos).

Esses dados encontram-se na direção contrária ao cenário em saúde mental no país. Entre os países em desenvolvimento, o Brasil é o primeiro do ranking mundial de prevalência da depressão, segundo levantamento nacional por amostragem (Andrade, Walter, Gentil, \& Laurenti, 2002). Os resultados do levantamento indicaram que $45,9 \%$ da população apresentou o diagnóstico de um transtorno mental durante a vida. Adicionalmente, esses achados parecem indicar um descompasso entre as demandasderivadas das políticas públicas acerca de programas preventivos baseados em evidências de eficácia e efetividade (Tykanori et al., 2013) e a atual produção acadêmica nesse campo. Esse diálogo entre políticas públicas e academia poderia ser fortalecido com um aumento do investimento em pesquisas de avaliação de eficácia e efetividade de programas preventivos.

Deve-se salientar que parte da produção da área está vinculada à promoção em saúde mental, cuja ênfase recai sobre a promoção de competências em saúde mental. Pode ser observada uma confusão conceitual entre os termos promoção de saúde, prevenção e tratamento. Tal confusão é respaldada pela literatura, que sugere um modelo integrativo conectando os três termos (Weisz, Sandler, Durlak, \& Anton, 2005). Nesse modelo, a sobreposição entre os termos se apresenta como algo compreensível, visto que a diferença conceitual entre eles é tênue. Tal sobreposição levanta a discussão sobre a promoção como uma condição para a prevenção e vice-versa. A grande diferença entre os 
Figura 2. O ciclo da pesquisa em prevenção no Brasil

\begin{tabular}{|c|c|c|}
\hline Etapas & Estudos Brasileiros & Ano de publicação \\
\hline $\begin{array}{l}\text { 1. Identificar o } \\
\text { problema e sua } \\
\text { prevalência }\end{array}$ & $\begin{array}{l}\text { Estudos teóricos e conceituais: } 43 \text { artigos } \\
\text { Pesquisa básica: } 32 \text { artigos } \\
\text { Epidemiológico: } 57 \text { artigos }\end{array}$ & $\begin{array}{l}\text { 1990-1999: } 16 \text { artigos } \\
\text { 2000-2005: } 25 \text { artigos } \\
\text { 2006-2011: } 91 \text { artigos }\end{array}$ \\
\hline
\end{tabular}

\begin{tabular}{|l|l|l|l|}
\hline $\begin{array}{l}\text { 2. Identificar fatores } \\
\text { de risco e proteção }\end{array}$ & Estudos de fatores de risco e proteção: 56 artigos & $\begin{array}{l}\text { 1990-1999: } 2 \text { artigos } \\
\text { 2000-2005: } 9 \text { artigos } \\
2006-2011: 45 \text { artigos }\end{array}$ \\
\hline
\end{tabular}

\begin{tabular}{|l|l|l|}
\hline $\begin{array}{l}\text { 3. Estudo piloto } \\
\text { baseado na teoria }\end{array}$ & $\begin{array}{l}\text { Programas com avaliação sistemática não experimentais : } 6 \\
\text { Avaliação de necessidades: 3 artigos } \\
\text { Criação de material de intervenção (cartillhas, jogos, manuais): } 5\end{array}$ & 1990-1999: 2 artigos \\
& $\begin{array}{l}\text { Desenvolvimento e avaliação de instrumentos de medida: } 6 \\
\text { 2000-2005: } 5 \text { artigos } \\
\text { Relato de experiência: 6 artigos } \\
\text { Descrição da implantação de programa: 4 artigos } \\
\text { Descrição de políticas de prevenção sem avaliar resultado: } 4\end{array}$ & \\
& & \\
\hline
\end{tabular}

\begin{tabular}{|l|l|l|l|}
\hline 4. Teste & $\begin{array}{l}|l| \\
\text { Programas com delineamento quase -experimental: } 3\end{array}$ & $\begin{array}{l}\text { 2000-2005: } 2 \text { artigos } \\
\text { Programas com delineamento experimental: } 1 \text { artigo }\end{array}$ & \\
\hline
\end{tabular}

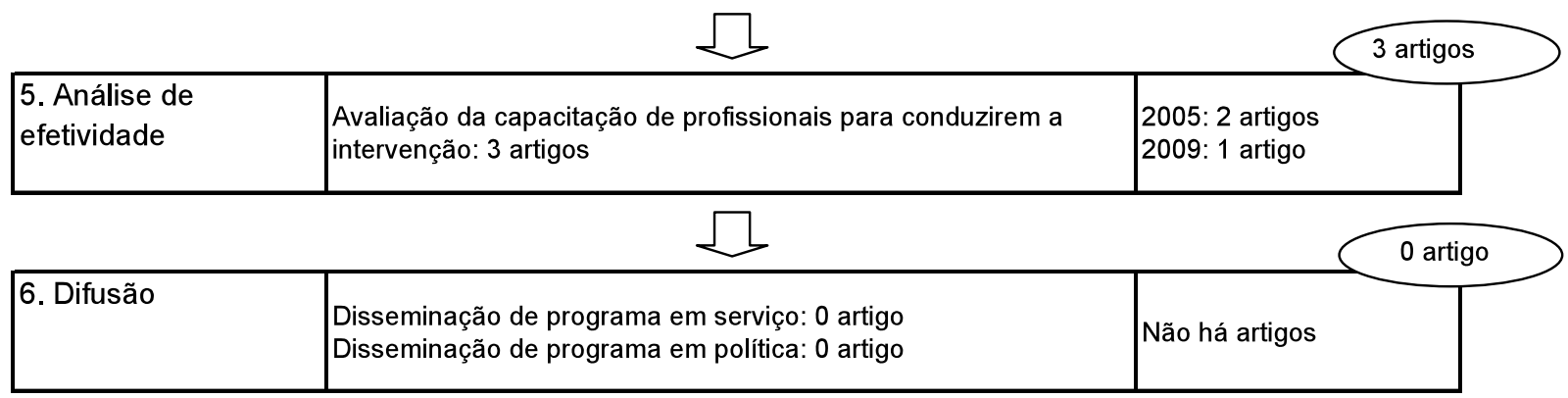

programas de prevenção e promoção estaria na avaliação de seus resultados. Há condições comuns sendo promovidas. Entretanto, os programas de promoção se detêm na avaliação da competência desenvolvida, enquanto os programas de prevenção medem os desfechos negativos que foram prevenidos a partir da redução em seus fatores de risco e da promoção de seus fatores de proteção (Weisz, Sandler, Durlak, \& Anton, 2005). Há evidências de que as intervenções preventivas mais eficazes são aquelas que atuam, simultaneamente, promovendo competências (fatores de proteção) e minimizando vulnerabilidades (fatores de risco) para um dado desfecho negativo (Cowen, 2000). Dessa forma, o desenvolvimento de medidas de avaliação compartilhadas entre as áreas de pesquisa de prevenção e promoção de saúde seria útil ao avanço de ambas.

A diminuição significativa de publicações à medida que o grau de complexidade das etapas do ciclo de pesquisa avança foi observada também em revisões de literatura dos programas preventivos nacionais (Canoletti \& Soares, 2004; Murta, 2005). Estudos nas etapas finais do ciclo de pesquisa em prevenção são relativamente mais complexos do que os das etapas iniciais e demandam formação profissional especializada, bem como recursos humanos e financeiros mais vultosos. É provável que lacunas na capacitação profissional e a insuficiência de fomento à 
pesquisa sejam algumas das razões que contribuem para tal cenário.

Avaliações sistemáticas de programas de prevenção a fatores de risco e transtornos em saúde mental, por meio de delineamentos robustos e instrumentos adequados, são cruciais para o avanço da área. Como consequência, seria facilitada a difusão de programas em larga escala para a comunidade. Nos Estados Unidos, por exemplo, órgãos públicos federais têm custeado a difusão de programas preventivos baseados em evidências, em detrimento de programas que não foram ainda suficientemente testados (Tibbits et al., 2010). A relevância da difusão de programas é contrária ao dado de que mais de $80 \%$ do que tem sido produzido no Brasil são estudos exploratórios e de fundamentação, acrescido da ausência de estudos de difusão. Entretanto, a mudança desse cenário é complexa, requerendoo apoio de políticas científicas específicas, bem como o fortalecimento do ensino da prática e pesquisa em prevenção, seja em cursos de graduação ou pós-graduação.

Os resultados deste estudo devem ser vistos com cautela, uma vez que foram analisados, exclusivamente, artigos científicos divulgados em bases de dados de grande circulação nacional e priorizada por profissionais da psicologia. Sugere-se que uma revisão mais ampla inclua teses, dissertações, anais de congresso, periódicos não indexados às bases de dados SciELO e PePSIC, capítulos de livros e artigos publicados em revistas internacionais. Para as publicações em inglês, sugere-se a busca pelo Currículo Lattes dos pesquisadores identificados neste estudo como fonte adicional de material de análise.

Diversas implicações resultam dos achados deste estudo para apesquisa em prevenção em saúde mental. A criação de uma rede de pesquisadores poderia favorecero desenvolvimento de programas preventivos, a condução de avaliações robustas e a disseminação entre contextos. Grupos multidisciplinares, com colaboradores da psicologia, saúde pública, saúde coletiva, serviço social, educação e gestão em políticas públicas, seriam especialmente frutíferos, uma vez que a área é eminentemente interdisciplinar. Igualmente recomendável é a aproximação entre a pesquisa em prevenção e a em promoção de saúde mental, dada a sobreposição entre seus métodos de intervenção, como os programas de desenvolvimento de habilidades sociais, que podem ser também utilizados em programas preventivos. É possível que o incremento de colaborações com grupos nacionais e internacionais estimule o progresso dos estudos em programas já desenvolvidos, como os relativos a prevenção a comportamento antissocial, rejeição por pares, violência, abuso de álcool e drogas e problemas emocionais e comportamentais, além de encorajar novos alvos de interesse, ainda não contemplados nos estudos ora identificados. Por certo, a colaboração entre grupos vinculados a centros de pesquisa nacionais e internacionais impactaria positivamente não somente a pesquisa, mas também o ensino, nos níveis de graduação e pós-graduação (Bianco, Almeida, Koller, \& Paiva, 2010).

Por fim, tem-se o grande desafio de inserção da produção da academia nas políticas públicas. A produção científica pode ter um papel mais ativo como fonte de informação para tomada de decisão de gestores e governantes sobre a natureza das políticas públicas de prevenção. Para tanto, a divulgação dos resultados de pesquisa deve ser acessível e convidativa, para que possa ser compreensível aos gestores e, quando disseminada, identificada como útil pela população. Nesse sentido, percebe-se, mais recentemente, um movimento conduzido pelos grandes órgãos científicos, de apoio à difusão do conhecimento científico. A divulgação de pesquisas em mídias não científicas vem sendo estimulada pelo Conselho Nacional de Desenvolvimento Científico e Tecnológico (CNPq), em uma atitude inovadora. $\mathrm{O}$ órgão incluiu a divulgação de pesquisas em meios populares de comunicação na avaliação de produtividade de pesquisadores, incluindo um espaço de registro da divulgação no sistema Lattes (Escola Nacional de Saúde Pública Sergio Arouca, 2012).

Esforços para a concretização dessa agenda, por parte de pesquisadores e gestores, estão diretamente associados à promoção de justiça social (Kenny \& Hage, 2009), um dos princípios norteadores da ciência da prevenção. Recursos públicos investidos na produção de conhecimento devem voltar à população, por meio de serviços que previnam o sofrimento psíquico grave e promovam bem-estar. Como já apontado por outros pesquisadores da área (HuynhNhu, Muñoz, Ippen \& Stoddard, 2003), esperar que as pessoas adoeçam para se fazer algo é condenar milhões ao sofrimento desnecessário. À comunidade científica, aos gestores $\mathrm{e}$ aos órgãos de fomento cabe $\mathrm{o}$ investimento em políticas científicas que incentivem estudos de eficácia, efetividade e difusão de programas de prevenção e promoção de saúde mental. Espera-se 
que eventuais esforços nesse sentido possam fazer avançar os estudos no ciclo de pesquisa em prevenção, para além da descrição de problemas em saúde mental e reconhecimento de seus fatores de risco e proteção, chegando a pesquisas para desenvolvimento, avaliação e disseminação de programas preventivos. Dessa forma, poder-se-á contribuir para que os anseios da sociedade referentes ao acesso a serviços de atenção básica e promoção de saúde mental (Sistema Único de Saúde, Conselho Nacional de Saúde, \& Comissão Organizadora da IV Conferência Nacional de Saúde Mental, 2010) sejam efetivamente atendidos.

\section{REFERÊNCIAS}

Andrade, L., Walters, E. E., Gentil V., \& Laurenti R. (2002). Prevalence of ICD-10 mental disorders in a catchment area in the city of Sao Paulo, Brazil. Social Psychiatry and Psychiatric Epidemiology, 7, 316-25.

Bardin, L. (1977). Análise de conteúdo. Lisboa: Edições 70.

Benetti, S. P. C., Ramires, V. R. R. Schneider, A. C., Rodrigues, A. P. G., \& Tremarin, D. (2007). Adolescência e saúde mental: revisão de artigos brasileiros publicados em periódicos nacionais. Cadernos de Saúde Pública, $23,1273-1282$.

Bolsoni-Silva, A. T., Silveira, F. F., \& Ribeiro, D. C. (2008). Avaliação dos efeitos de uma intervenção com mães/ cuidadoras: contribuições do treinamento em habilidades sociais. Contextos Clínicos, 1, 19-27.

Borges, D. S. C., \& Marturano, E. M. (2009). Aprendendo a gerenciar conflitos: um programa de intervenção para a $1^{\mathrm{a}}$ série do ensino fundamental. Paidéia, 19, 17-26.

Brasil, Ministério da Saúde, Departamento de Ações Programáticas Estratégicas, Coordenação de Saúde Mental (2003). O vínculo e o diálogo necessários inclusão das ações de saúde mental na atenção básica. Brasília: Ministério da Saúde.

Brasil, Ministério da Saúde, Departamento de Ações Programáticas Estratégicas, Coordenação de Saúde Mental (2006). Saúde mental em dados 3. Brasília: Ministério da Saúde.

Brasil, Ministério do Desenvolvimento Social e Combate à Fome (2012). Orientações técnicas sobre o PAIF. Brasília: MDS, Secretaria Nacional de Assistência Social.

Brasil, Ministério da Saúde, Secretaria de Atenção à Saúde (2008). Política Nacional de Atenção Integral à Saúde do Homem. Brasília: Secretaria de Atenção à Saúde.

Canoletti, B.,\& Soares. C. B. (2004) Programas de prevenção ao consumo de drogas no Brasil: uma análise da produção científica de 1991 a 2001. Interface - Comunicação, Saúde e Educação, 16, 115-129.

Castro, R. E. F., Melo, M. H. S., \& Silvares, E. F. M. (2003). $O$ julgamento de pares de crianças com dificuldades interativas após um modelo ampliado de intervenção. Psicologia: Reflexão e Crítica, 16, 309-318.

Christensen, H., Pallister, E.,Smale, S., Hickie, I. B., \& Calear, A. L. (2010). Community-based prevention programs for anxiety and depression in youth: a systematic review. Journal of Primary Prevention, 31, 139-170.

Escola Nacional de Saúde Pública Sergio Arouca (2012). Ajustes na Plataforma Lattes estimulam a divulgação científica. Informe ENSP, 1-2. Retirado de: http://www.ensp.fiocruz.br/portal-

ensp/informe/site/materia/detalhe/29770

Cowen, E. L. (2000). Now that we all know that prevention in mental health is great, what is it? Journal of Community Psychology, 28, 5-16.

Cuijpers, P., Straten, A., Smit, F., Mihalopoulos C., \& Beekman, A. (2008).Preventing the onset of depressive disorders: a meta-analytic review of psychological interventions. American Journal of Psychiatry, 10, 12721280.

De Micheli, D., Fisberg, M., \& Formigoni, M. L. O. S (2004). Estudo da efetividade da intervenção breve para o uso de álcool e outras drogas em adolescentes atendidos num serviço de assistência primária à saúde. Revista da Associação Médica Brasileira, 50, 305-313.

Doughty, C. (2005). The effectiveness of mental health promotion, prevention and early intervention in children, adolescents and adults: a critical appraisal of the literature. New Zealand Health Technology Assessment Report, 8, 7-12.

Dumka, L. E., Rosa, M. W., Michaels, M. L., \& Suh, K. W. (1995). Using research and theory to develop prevention program for high-risk families. Family Relations, 44, 7886.

Durlak, J. A., \& Wells, A. M. (1997). Primary prevention mental health programs for children and adolescents: a meta-analytic review. American Journal of Community Psychology, 25, 115-142.

Fisak Jr, B., Richard, D., \& Mann, A. (2011). The prevention of child and adolescente anxiety: a meta-analytic review. Prevention Science, 12, 255-268.

Flay, B. R., Biglan, A., Boruch, R., Castro, F. G., Gottfredson, D., Kellam, S., Moscicki, E. K., Schinke, S., Valentine, J. C., \& Ji, P. (2005). Standars of evidence: criteria for efficacy, effectiveness and dissemination. Prevention Science, 6, 151-175.

Huynh-Nhu, L., Munõz, R. F., Ippen, C. G., \& Stoddard, J. L. (2003). Treatment is not enough: we must prevent major depression in women. Prevention \& Treatment, 6 .

Kazdin, A. E. (1982). Methods for clinical and applied settings: New York: Oxford University Press.

Kenny, M. E. \& Hage, S. M. (2009). The next frontier: prevention as an instrument of social justice. The Journal of Primary Prevention, 30, 1-10.

Knnerr, W., Gardner, F., \& Cluver, L. (2013). Improving positive and parenting skills and reducing rash and abusive parenting in low-and middle income countries: a systematic review. Prevention Science, 14, 352-363.

Knapp, M., McDaid, D., \& Parsonage, M. (2011). Mental health promotion and mental health illness prevention: the economic case. London: Department of Health.

Lacerda Júnior, F., \& Guzzo, R. S. L. (2005). Prevenção primária: Análise de um movimento e possibilidades para 
o Brasil. Interação em Psicologia, 9, 239-249.

Lo Bianco, A. C., Almeida, S. S., Koller, S. H., \& Paiva, V. (2010). A internacionalização dos programas de pósgraduação em psicologia: perfil e metas de qualificação. Psicologia: Reflexão e Crítica, 23, 1-10.

Lohr, S. S., Pereira, A. C. S., Andrade, A. L. M., \& Kirchner, L. F. (2007). Avaliação de programas preventivos: relato de experiência. Psicologia em Estudo, 12, 641-649.

Louzã, M. R. (2007). Detecção precoce: é possível prevenir a esquizofrenia? Revista de Psiquiatria Clínica, 34, 169173.

Luizzi, L.,\& Rose, T. M. S. (2010). Intervenções para a prevenção e redução de comportamentos agressivos e a formação de professores. Temas em Psicologia, 18, 5769.

Melnik, T. \& Atallah, A. N. (2011). Psicologia baseada em evidências: articulação entre a pesquisa e prática clínica. Em T. Menik \& A. N. Atallah (Org.), Psicologia baseada em evidências: provas científicas da efetividade da psicoterapia (pp. 3-13). São Paulo: Santos.

Muñoz, R. F., Cuijpers, F., Smit, F., Barrera, A. Z., \& Leykin, Y. (2010). Preventionof major depression. Annual Review of Clinical Psychology, 6, 181-212.

Murta, S. G. (2005). Aplicações do treinamento em habilidades sociais: análise da produção nacional. Psicologia. Reflexão e Crítica, 18, 283-291.

Murta, S. G. (2007). Programas de prevenção e problemas emocionais e comportamentais em crianças e adolescentes: lições de três décadas de pesquisa. Psicologia: Reflexão e Crítica, 20, 1-8.

Murta, S. G., Borges, F. A., Ribeiro, D. C., Rocha, E. P., Menezes, J. C. L., \& Prado, M. M. (2009). Prevenção primária em saúde na adolescência: Avaliação de um programa de habilidades de vida. Estudos de Psicologia (UFRN), 14, 181-189.

Murta, S. G., Santos, B. R. P., Martins, C. P. S., \& Oliveira, B. (2013). Prevenção à violência no namoro: uma revisão de literatura. Contextos Clínicos, 6, 117-131.

Pelisoli, C., \& Picoloto, L. B. (2010). Prevenção do abuso sexual infantil: estratégias cognitivocomportamentais na escola, na família e na comunidade. Revista Brasileira de Terapias Cognitivas, 6, 108-137.

Pinheiro, M. I. S., Haase, V. G., Del Prette, A., Amarante, C. L. D., \& Del Prette, Z. A. P. (2006). Treinamento de habilidade sociais educativas para pais de crianças com problemas de comportamento. Psicologia: Reflexão $e$ Crítica, 19, 407-414.
Prada, C, G., Williams, L. C. A., \& Weber, L. N. D. (2007). Abrigos para crianças vítimas de violência doméstica: funcionamento relatado pelas crianças e pelos dirigentes. Psicologia: Teoria e Prática , 9, 14-25.

Rios, K. S. A.,\& Williams, L. C. A. (2008). Intervenção com famílias como estratégia de prevenção de problemas de comportamento em crianças: uma revisão. Psicologia em Estudo, 13, 799-806.

Rodrigues, M. C., Dias, J. P., \& Freitas, M. F. R. L. (2010). Resoluções de problemas interpessoais: promovendo o desenvolvimento sociocognitivo na escola. Psicologia em Estudo, 15, 831-839.

Ronzani, T. M., Rodrigues, T. P, Batista, A. G., Lourenço, L. M., \& Formigoni, M. L. O. S. (2007). Estratégias de rastreamento e intervenções breves para problemas relacionados ao uso de álcool entre bombeiros. Estudos de Psicologia, 12, 285-290.

Silva, J. M. A. P.,\& Salles, L. M. F (2010). A violência na escola: abordagens teóricas e propostas de prevenção. Educar em Revista, 2, 217-232.

Sistema Único de Saúde, Conselho Nacional de Saúde, Comissão Organizadora da IV Conferência Nacional de Saúde Mental - Intersetorial (2010). Relatório Final da IV Conferência Nacional de Saúde Mental - Intersetorial. Brasília: Conselho Nacional de Saúde/Ministério da Saúde.

Tibbits, M. K., Bumbarger, B. K., Kyler, S. J., \& Perkins, D. F. (2010). Sustaining evidence-based interventions under real-world conditions: results from a large-scale diffusion project. Prevention Science, 11, 252-262.

Tykanori, R., Santos, N., Pedroso, R. T., Abreu, S., Rabbani, A. R., Rocha, V., Schneider, D., Sanchez, Z., Atayde, R., Medeiros, P. F. P., \& Scafuto, J. (2013). Adaptation and evaluation of drug abuse prevention programs as a Brazilian public policies. Trabalho apresentado na 4th International Conference and Members' Meeting of European Society for Prevention Research. Paris: European Society for Prevention Research.

Weisz, J. R., Sandler, I. N., Durlak, J. A., \& Anton, B. S. (2005). Promoting and protecting youth mental health through evidence-based prevention and treatment. American Psychologist, 60, 628-648.

Recebido em: 17/12/2013 Primeira decisão editorial em: 18/12/2014 Aceito em: 22/01/2016

Notas:

A primeira autora agradece ao CNPq pela concessão de bolsa para Mestrado, realizado no Programa de PósGraduação em Psicologia Clínica (UnB), sob a orientação da segunda autora.

Agradecimentos a Marina Pedralho pelo auxílio na coleta e categorização dos dados. 